\title{
Role of social network services (SNS) sales promotions in generating brand loyalty for chain steakhouses
}

\begin{abstract}
This research aimed to explore the impact of social network services (SNS) sales promotions on the brand loyalty generation process by considering the role of brand attitude, awareness, service quality, experience, trust, and attachment in the chain steakhouse industry. A field survey was conducted to collect data in a metropolitan city of South Korea. According to our structural equation modeling results, SNS sales promotions significantly increased loyalty and its antecedents, and brand attachment had the greatest influence on brand loyalty. The mediating impact of study variables was also identified. The proposed model had a satisfactory level of prediction power for loyalty. Overall, our findings provided important insights into how steakhouse practitioners can deal with SNS as essential promotional tools and provided valuable insights into why using SNS sales promotions is critical in the fastchanging chain restaurant industry.
\end{abstract}

Keyword: Chain steakhouse industry; Social network services; Sales promotions; Brand loyalty; Brand attachment; Restaurant customers 